\title{
Movement-Related Potentials Associated with Motor Timing Errors as Determined by Internally Cued Movement Onset
}

\author{
Jee Seon Ahn',2, Jun Ho Yoon ${ }^{3}$, Jae-Jin Kim²,4, and Jin Young Park²,5, $₫$ \\ ${ }^{1}$ Graduate Program in Cognitive Science, Yonsei University, Seoul, Republic of Korea \\ ${ }^{2}$ Institute of Behavioral Science in Medicine, Yonsei University College of Medicine, Yonsei University Health System, Seoul, Republic of Korea \\ ${ }^{3}$ Department of Neurology, Seoul National University College of Medicine, Seoul National University Bundang Hospital, Seongnam, \\ Republic of Korea \\ ${ }^{4}$ Department of Psychiatry, Yonsei University College of Medicine, Gangnam Severance Hospital, Yonsei University Health System, Seoul, \\ Republic of Korea \\ ${ }^{5}$ Department of Psychiatry, Yonsei University College of Medicine, Yongin Severance Hospital, Yonsei University Health System, Yongin, \\ Republic of Korea \\ ${ }^{6}$ Center for Digital Health, Yongin Severance Hospital, Yonsei University Health System, Yongin, Republic of Korea
}

Objective Accurate motor timing is critical for efficient motor control of behaviors; however, the effect of motor timing abilities on movement-related neural activities has rarely been investigated. The current study aimed to examine the electrophysiological correlates of motor timing errors.

Methods Twenty-two healthy volunteers performed motor timing tasks while their electroencephalographic and electromyographic (EMG) activities were simultaneously recorded. The average of intervals between consecutive EMG onsets was calculated separately for each subject. Motor timing error was calculated as an absolute discrepancy value between the subjects' produced and given time interval. A movement-related potential (MRP) analysis was conducted using readings from $\mathrm{Cz}$ electrode.

Results Motor timing errors and MRPs were significantly correlated. Our principal finding was that only Bereitschaftpotential (BP) and motor potential (MP), not movement monitoring potential, were significantly attenuated in individuals with motor timing errors. Motor timing error had a significant effect on the amplitude of the late BP and MP.

Conclusion The findings provide electrophysiological evidence that motor timing errors correlate with the neural processes involved in the generation of self-initiated voluntary movement. Alterations in MRPs reflect central motor control processes and may be indicative of motor timing deficits.

Psychiatry Investig 2021;18(7):670-678

Key Words Time perception, Psychomotor performance, Motor activity, Electroencephalography, Electromyography.

\section{INTRODUCTION}

Timing ability is a core aspect facilitating human behavior at both the motor and perceptive levels. ${ }^{1}$ Adequate timing of behavior is not possible without temporal perception, and the temporal organization of motor output is greatly dependent on the representation of time in the brain. ${ }^{2}$ A self-initiated voluntary movement is preceded by a period of neural activity be-

\footnotetext{
Received: December 16, 2020 Revised: April 7, 2021

Accepted: April 18, 2021

$\triangle$ Correspondence: Jin Young Park, MD, PhD

Department of Psychiatry, Yonsei University College of Medicine, Yongin Severance Hospital, 363 Dongbaekjukjeon-daero, Giheung-gu, Yongin 16995, Republic of Korea

Tel: +82-31-5189-8148, Fax: +82-31-5189-8565, E-mail: empathy@yuhs.ac

(c) This is an Open Access article distributed under the terms of the Creative Commons Attribution Non-Commercial License (https://creativecommons.org/licenses/bync/4.0) which permits unrestricted non-commercial use, distribution, and reproduction in any medium, provided the original work is properly cited.
}

ginning up to 1 second or longer before the onset of voluntary movements and is maximal over the central-midline cortical areas. ${ }^{3}$ This activity, termed "movement-related potentials" (MRP), is probably generated by a summation of excitatory postsynaptic potentials in apical cortical dendrites. Electroencephalographic (EEG) has a high level of temporal resolution and is sensitive to the timing of neuronal synaptic and dendritic activity. Larger negativity recorded over the scalp indicates larger activity, reflecting a greater amount of depolarization in the apical dendrites of pyramidal neurons. ${ }^{4}$

MRPs are the EEG evidence of cortical involvement during the planning, preparation, execution, and evaluation of movements. ${ }^{5}$ In motor tasks used to elicit MRPs, what subjects should actually do is to execute the same movements repeatedly. Like all event-related potentials, the motor-related potential is measured as the average of the potentials from repetitions of the 
same event. ${ }^{6}$ the EEG signals from each trial are time-locked to the onset of voluntary muscle contractions and averaged to reduce noise. ${ }^{3,5}$ MRPs consist of Bereitschaftpotential (BP), a negative cortical potential that develops slowly from 1 second or longer before electromyographic (EMG) onset; the motor potential (MP) with peak negativity; and the movement monitoring potential (MMP) to rebound again. ${ }^{3,7}$ Several past studies show the contribution from the supplementary motor area (SMA) to this potential. The amplitude of the BP over midline central structures and amount of active SMA neurons are increased during internally driven motor acts. The BP prior to incidental or externally triggered movements in early midline central neural activity was absent. This suggests that BP is correlated specifically with voluntary action. ${ }^{8}$

Neuro-cognitive models of time estimation have shown SMA and corticostriatal circuits to be the neuronal substrates of an internal clock that creates a representation of time on which mechanisms of voluntary movement initiation rely. ${ }^{9}$ The motor circuit including the SMA is also related to dopamine levels. These brain systems guide the estimation of time intervals and the timing of movement. Since interval timing deficits have been observed in neuropsychiatric diseases, interval timing is considered a fundamental cognitive component. Examples of such psychiatric conditions with dopaminergic dysfunctions include Parkinson's disease, ${ }^{10-12}$ schizophrenia, ${ }^{13}$ attention-deficit hyperactivity disorder, ${ }^{14,15}$ autism spectrum disorder ${ }^{16}$ and Gilles de la Tourette syndrome. ${ }^{17}$ Motor timing could be explained as a fundamental aspect of the temporal organization of behavior, ${ }^{15,18}$ and a deficit in time perception might impact motor output and, consequently, motor skills. ${ }^{18}$

In an experimental setting, voluntary movements are the absence of any external trigger. If the physical manifestation of the behavior is prespecified and kept constant throughout the experiment, the most likely behavior to be identified differently by the subjects will be the motor timing. ${ }^{19}$ In contrast to reflex action by external triggers, internal time processing is essential for voluntary control of movement, and time intervals should be internally generated between voluntary movements. Selfinitiated voluntary movements are accompanied by subjective temporal perception and motor timing skills. ${ }^{20,21}$ Perceptions of duration are scaled according to each subject's internal clock. Subjective time is the internal experience of how fast time is passing, or how much time has passed since the occurrence of some event. Motor timing refers to the ability to withhold movement voluntarily until the right moment to generate a correct time interval that equates to the output of behavior. ${ }^{22}$

Various researchers have attempted to examine purely motoric or cognitive processes that contribute to the planning and preparation of self-initiated voluntary movements. ${ }^{9,19,23,24}$ However, much focus has been placed to date on the BP and the motor timing process contributing to the entire MRP activity has not been studied comprehensively, despite its great importance in self-initiated voluntary movements. It is worthwhile to explore MRPs in the temporal control of behavior for a fundamental understanding of the neural mechanisms in motor timing processes. Therefore, it is necessary to establish the relative contribution of motor timing processes to the MRPs. We expected that central-midline activity, particularly the SMA underlying $\mathrm{Cz}$, will reflect internal generation of motor timing. We hypothesized that there will be a greater activity when accurately fit into the specified temporal range. The aim of this preliminary study was to investigate neural activity related to motor timing processes in healthy subjects.

\section{METHODS}

\section{Subjects}

The present study was conducted from July 2019 to April 2020 in a university-affiliated hospital (Severance Hospital, Seoul, Korea). Twenty-two healthy volunteers participated in the experiment. All subjects were part of an ongoing study concerning Tourette's disorder and motor tic disorder. Subjects were excluded if they met any of the following criteria: were younger than 18 years old, had brain lesions or a history of neurological or psychiatric disorders, used psychiatric medications, or were deemed unable to participate in the study by a psychiatrist. The 22 [17 (77.3\%) female] subjects included in this investigation, the mean [ \pm standard deviations (SDs)] age ranged from 19 to 58 years $(33.1 \pm 12.7$ years). All subjects were righthanded as assessed by the Edinburgh handedness inventory. Each subject provided written, informed consent to participate in the study after being informed about the purpose and procedures involved. The Institutional Review Board of Severance Hospital, Yonsei University, reviewed and approved this study (no. 4-2019-0165).

\section{Procedures}

Each subject sat comfortably in a chair with their forearm placed on an armrest in a quiet room. Subjects were instructed to initiate a new brisk movement with their right wrist repeatedly as closely as possible to the specified time interval ( 5 seconds) without specifying the total number of movements. A total of 1,395 trials were included in this investigation, with the subjects ranging from 39 to 99 trials in 5 minutes and the mean $( \pm S D)$ of $63.41 \pm 15.51$.

This was completed by a personal judgement of the temporal range without the use of any external time device. Before the task began, a brief warm-up round was conducted. Scalp EEG and surface EMG data were simultaneously recorded while subjects performed the instructed task. During the recording, 
to minimize eye movement, subjects were asked to focus on a fixation mark straight ahead with their eyes open and jaw musculature relaxed. The total duration for the completion of the tasks was approximately 30 minutes, with two breaks of approximately 5 minutes each offered. The importance and direct attention to the timing of the movement were explained to the subjects. The instructions were given verbally to the subjects as follows:

1) Rest your forearm on the armrest with your hand hanging downward. Face your palm downward without stretching your fingers. Keep your fingers relaxed. Fix your eyes on the fixation mark straight ahead while minimizing eye-related and jaw movement to prevent movement-related artifacts in the recording.

2) Extend your dominant wrist as briskly as possible, and then relax your hand muscles as quickly as possible (drop the hand below gravity back to its resting position) to avoid inducing any muscular tension, which could possibly affect the outcome. Extend the wrist with a moderate force to avoid muscular tiredness.

3) The interval between self-initiated wrist extensions should be approximately 5 seconds. Achieve a regular interval between two successive movements. It is important to comply with the temporal intervals and the time to movement onset.

4) The arm and hand muscles should be completely relaxed during the interval between movements. Eye blinks, body adjustments, throat clearing, and other movements should be avoided. Avoid blinking for as long as possible.

5) A brief practice session is performed to help you understand the task and enable brisk right movements. You may be interrupted during the practice session to ask if you are able to follow the instructions correctly. Short pauses are allowed during a task upon request or the presence of many artifacts on the on-line recordings.

\section{Recording}

EMG activity was measured using $\mathrm{Ag} / \mathrm{AgCl}$ bipolar surface electrodes (HeartRode; HUREV Company, Wonju, Korea) placed over the right wrist extensor muscle (extensor digitorum muscle). The EMG data were digitized at $1 \mathrm{kHz}$ using the Physio 16 MR input box (Electrical Geodesics, Eugene, OR, USA) and filtered online using a bandpass filter set at 10-120 Hz. A 64-channel Hydrocel Geodesic Sensor Net (Electrical Geodesics, Eugene, OR, USA) was used to place electrodes on the scalp in accordance with the extended international 1020 system. EEG signals were amplified by the Net Amps 400 Amplifier (Electrical Geodesics, Eugene, OR, USA) and EEG data were continuously recorded with the Net station version 5.4 software (Electrical Geodesics, Eugene, OR, USA). The continuous EEG was sampled at $1 \mathrm{kHz}$. For all electrodes, the impedance was maintained below $50 \mathrm{kOhms}$. EEG data were band-pass-filtered at 0.5 to $100 \mathrm{~Hz}$ and notch-filtered at $60 \mathrm{~Hz}$. All EEG channels were referenced to the vertex $(\mathrm{Cz})$ of the scalp.

\section{Analysis}

\section{EMG analysis}

EMG data were exported and analyzed using MATLAB 2015b (MathWorks, Natick, MA, USA). The continuous EMG signal was rectified and then filtered using a second-order Butterworth filter; a cut-off frequency of $20 \mathrm{~Hz}$ was applied to smooth the features. The timing of EMG onset was defined as the point at which the EMG activity exceeded two SDs above the resting baseline in a manner that was maintained for at least $80 \mathrm{~ms}^{25}$ In each trial, EMG onset was determined both by visual inspection and by using computer-based onset determination for the smoothed EMG record. If the EMG onset could not be determined using any method, it was recorded as a missing value for the statistical analysis. ${ }^{26}$ The baseline shift was also corrected in the software afterward, defining the zero level from the trace. The amplitudes of the EMG and the latencies were measured with respect to the peak of the averaged rectified EMG activity.

\section{Behavior analysis}

To quantify the behavior performance during the motor timing task, inter-movement time was calculated as the average of all intervals between consecutive EMG onsets performed for each of the subjects for 5 minutes (Supplementary Figure 1 in the online-only Data Supplement). Since the subjects were instructed to extend their dominant wrist as actively as possible and then drop the hand under gravity to its resting position, we considered the EMG peak latency between the onset and peak of the average rectified EMG activity as movement duration instead of the EMG offset. Motor timing error was calculated as an absolute discrepancy value between the subjects' produced and given time interval ( 5 seconds). The motor timing errors were calculated ${ }^{14}$ as follows: motor timing error= the mean duration of intervals between consecutive EMG onsets - given time interval (5 seconds)|. This reflects the magnitude of the subjects' motor timing errors regardless of directionality (over- or underestimates).

\section{MRP analysis}

EEG data were preprocessed and analyzed using functions from the EEGLAB toolbox operating under MATLAB 2015b (MathWorks, Natick, MA, USA). ${ }^{27}$ The continuous EEG data were re-referenced to an average reference and filtered with a band-pass of 0.01 to $35 \mathrm{~Hz}$. EEG artifacts were removed by independent component analysis. A total of 126 ICA components were removed among the subjects. The number of ICA components removed per subject ranged from 3 to 9 with a 
mean $( \pm S D)$ of $5.73 \pm 1.96$ (Supplementary Table 1 in the online-only Data Supplement). MRP was derived from movement onset trigger-averaged EEG signals. The EMG onset was marked on the EEG recording's time axis $(t=0 \mathrm{~s})$. The continuous EEG data were segmented into $3 \mathrm{~s}$ epochs, including $2 \mathrm{~s}$ before to $1 \mathrm{~s}$ after with respect to EMG onset. Meanwhile, the baseline was defined as the time window from $-2 \mathrm{~s}$ to $-1.9 \mathrm{~s}$, referencing the $\mathrm{EMG}$ onset $(\mathrm{t}=0 \mathrm{~s})$. All trials were visually inspected. Epochs with excessive artifacts were rejected and the remaining epochs were then averaged. The mean $( \pm S D)$ number of trials left after data rejection was $57.8 \pm 12.9$ per subject. For the MRP, we measured the voltage at the central-midline channel, Cz. Based on data observation, restricted time windows were selected to identify specific components and measure the amplitude of these components. Four components of movement-related potentials (early BP, late BP, MP, and MMP) were considered: the early BP with a mean amplitude of $-1 \mathrm{~s}$ to $-0.5 \mathrm{~s}$; the late BP with a mean amplitude of $-0.5 \mathrm{~s}$ to $0 \mathrm{~s}$ (EMG onset); MP as the peak negativity and latency; MMP with a mean amplitude of $0 \mathrm{~s}$ to $0.5 \mathrm{~s}$. Measurements were made on the individual records. ${ }^{28-30}$

\section{Statistical analysis}

Statistical analyses were performed using the Statistical Package for the Social Sciences version 25.0 (IBM Corp., Armonk, NY, USA) software. All p-values were two-tailed and statistical significance was set at less than 0.05 . To determine the variables to be used in the regression model, Pearson's correlation coefficient was used to test the possible significant correlations between MRP measures and behavior characteristics. Relationships were determined using linear regression models to assess the associations between covariables of interest and MRP components. A linear regression analysis with the "enter" method was conducted to evaluate the independent determinants of each MRP component.

\section{RESULTS}

An overview of the results in subjects is depicted in Table 1 and the grand average waveforms of the MRPs recorded during the motor timing task for the subject is demonstrated in Figure 1. The plots also show the typical time course for MRPs from $\mathrm{Cz}$ electrodes. The slow negative potential began to increase around $1.5 \mathrm{~s}$ prior to EMG onset and reached a peak amplitude around the point of EMG burst. After the peak, the potential returned to the baseline level. According to the behavioral data, the mean $( \pm S D)$ for the total number of trials conducted by subjects in 5 minutes was $63.41 \pm 15.51$. The mean $( \pm \mathrm{SD})$ time interval between consecutive EMG onsets among the subjects was $5,911.2 \pm 1,233.1 \mathrm{~ms}$, and the mean $( \pm S D)$ for the motor timing errors was $1,092.7 \pm 1,067.4 \mathrm{~ms}$.

Pearson's correlation coefficients were used to establish the correlation between the MRP components and behavior characteristics regarding the motor timing task. As shown in Figure 2, a significant correlation was found between the motor timing error and amplitude of early $\mathrm{BP}(\mathrm{r}=0.485 ; \mathrm{p}=0.022)$, late $\mathrm{BP}(\mathrm{r}=0.5118 ; \mathrm{p}=0.015)$, and $\mathrm{MP}(\mathrm{r}=0.570 ; \mathrm{p}=0.006)$. There was no significance between the motor timing error and amplitude of MMP ( $r=0.381 ; \mathrm{p}=0.080)$. There was no correlation between EMG peak latency and MRP components (early BP, $r=0.396$, $\mathrm{p}=0.068$; late $\mathrm{BP}, \mathrm{r}=0.352$, $\mathrm{p}=0.108$; MMP, $\mathrm{r}=0.176 \mathrm{p}=0.434$ ). In contrast, MP showed a significant positive correlation with an EMG peak latency $(r=0.440, p=0.040)$. There were no significant correlations between EMG peak amplitude and MRP components (early BP, $\mathrm{r}=-0.188, \mathrm{p}=0.403$; late $\mathrm{BP}, \mathrm{r}=-0.203$, $\mathrm{p}=0.365$; MP, $\mathrm{r}=-0.79$, $\mathrm{p}=0.727$; MMP, $\mathrm{r}=-0155, \mathrm{p}=0.492$ ) (Table 2).

In the linear regression models, motor timing error was independently and positively associated with the late $\mathrm{BP}(\mathrm{p}=0.036)$ (Table 3$)$ and $\mathrm{MP}(\mathrm{p}=0.016)$ (Table 4$)$ but not with early BP $(\mathrm{p}=$ $0.056)$ and MMP ( $\mathrm{p}=0.360)$. EMG peak latency was not associated with MRP components: early BP $(\mathrm{p}=0.173)$, late BP ( $\mathrm{p}=$

Table 1. Behavior, and electrophysiological data of the study sample

\begin{tabular}{lrrrr}
\hline & Mean & SD & Min & Max \\
\hline Trials & 63.41 & 15.51 & 39.00 & 99.00 \\
Interval time $(\mathrm{ms})$ & $5,911.20$ & $1,233.14$ & $4,457.88$ & $9,069.19$ \\
Motor timing errors $(\mathrm{ms})$ & $1,092.69$ & $1,067.43$ & 24.37 & $4,069.19$ \\
EMG peak amplitude $(\mu \mathrm{V})$ & 174.19 & 60.06 & 71.89 & 321.74 \\
EMG peak latency $(\mathrm{ms})$ & 241.67 & 234.72 & 85.00 & 967.00 \\
Early BP $(\mu \mathrm{V})$ & -0.97 & 0.71 & -2.43 & 0.29 \\
Late BP $(\mu \mathrm{V})$ & -1.78 & 1.17 & -4.92 & 0.20 \\
MP $(\mu \mathrm{V})$ & -3.29 & 1.61 & -6.26 & -0.78 \\
MMP $(\mu \mathrm{V})$ & -1.43 & 1.18 & -3.59 & 0.32 \\
\hline
\end{tabular}

EMG: electromyogram, BP: Bereitschaftpotential, MP:motor potential, MMP: movement monitoring potential, SD: standard deviation, Min: minimum, Max: maximum 
$0.269), M P(p=0.114)$, and MMP ( $p=0.739)$. We found that a less accurate motor timing indicated attenuated the MRP amplitude. Motor timing error explained $34.8 \%$ of the peak am- plitude of MRP [adjusted $\mathrm{R}^{2}=0.348 ; \mathrm{F}(2,21)=6.594, \mathrm{p}=0.007$; sum of squares regression $=22.32$, residual $=32.15]$.

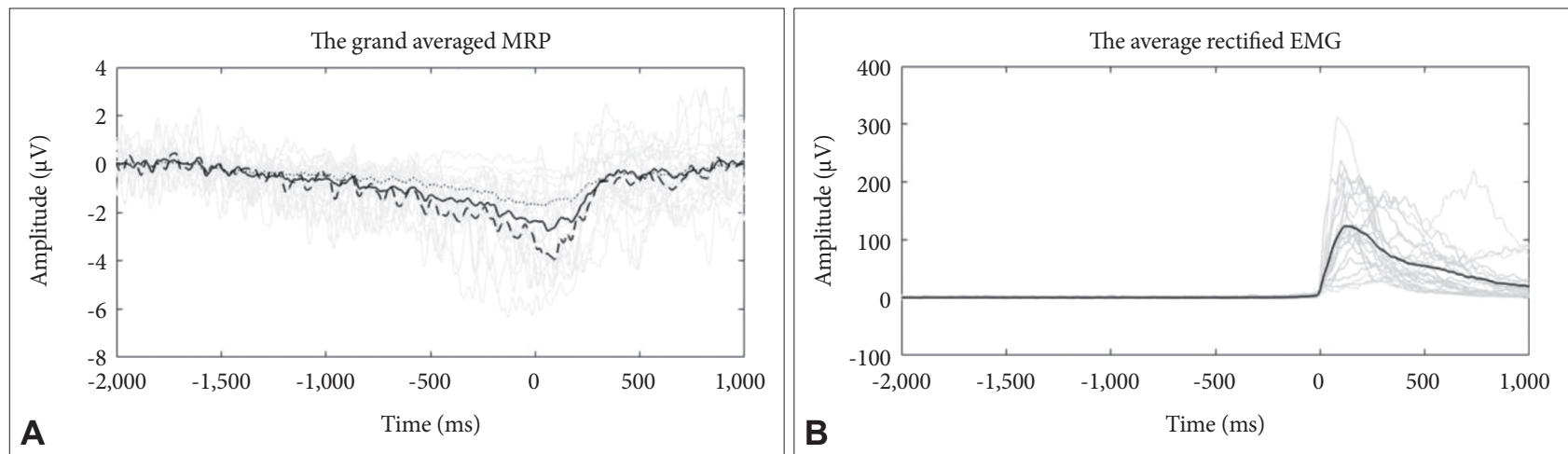

Figure 1. The average rectified EMG (B) and grand average MRP from $2 \mathrm{~s}$ before to $1 \mathrm{~s}$ after the EMG onset at selected electrode sites $(\mathrm{Cz})(\mathrm{A})$, showing typical morphology in study sample; over all subjects (solid line) subjects with motor timing errors in the first quintile (Q1) (dotted line) subjects with motor timing errors in the fifth quintile (Q5) (dashed line). Time 0 indicates EMG onset. MRP: movement-related potential, EMG: electromyogram.
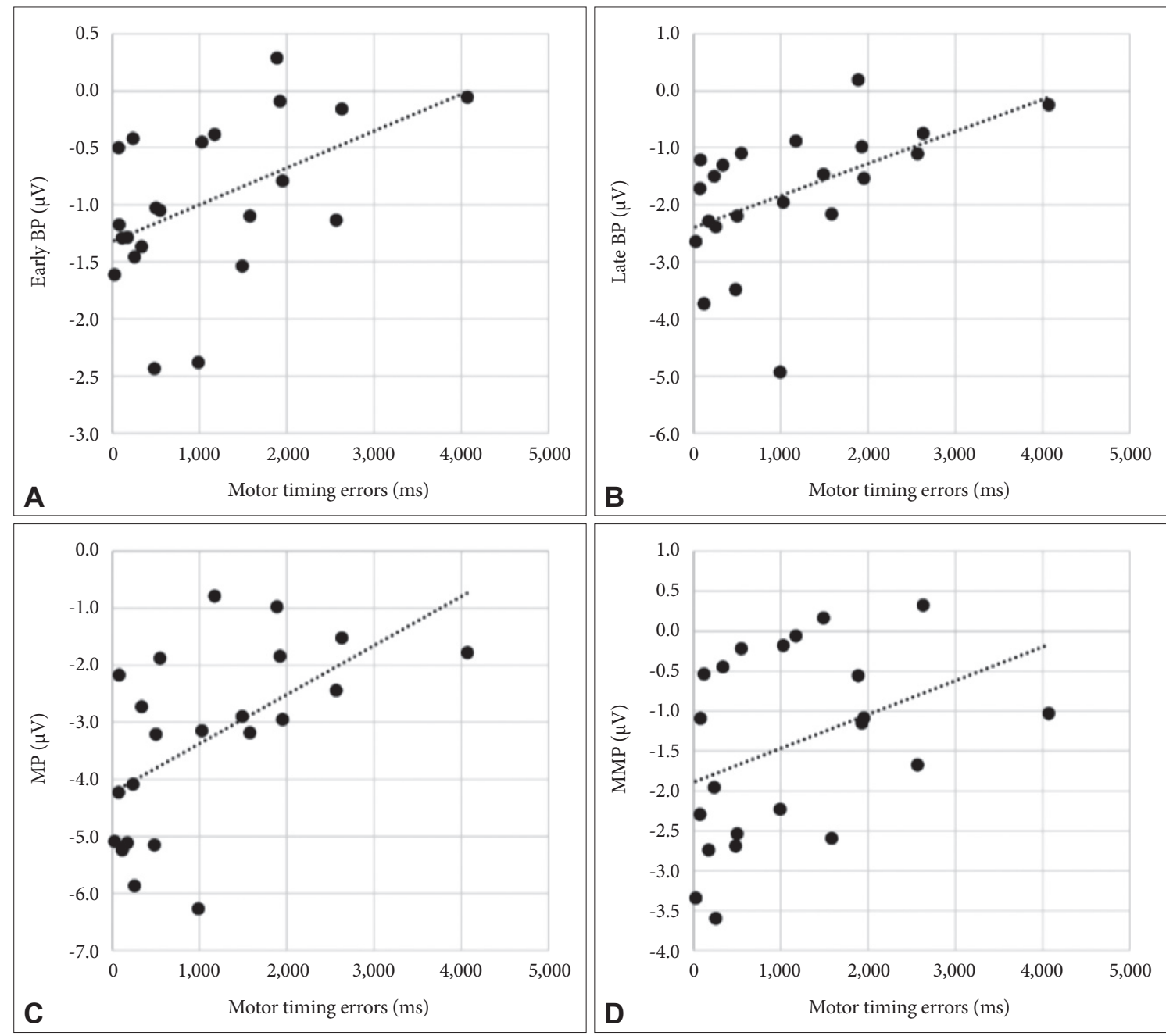

Figure 2. Correlation (linear fit) between the motor timing error and the early BP amplitude at $\mathrm{Cz}(\mu \mathrm{V})(\mathrm{r}=0.485$; $\mathrm{p}=0.022)(\mathrm{A})$; the late $\mathrm{BP}$ amplitude at $\mathrm{Cz}(\mu \mathrm{V})(\mathrm{r}=0.512 ; \mathrm{p}=0.015)(\mathrm{B})$; the MP amplitude at $\mathrm{Cz}(\mu \mathrm{V})(\mathrm{r}=0.570 ; \mathrm{p}=0.006)(\mathrm{C})$; the MMP amplitude at $\mathrm{Cz}(\mu \mathrm{V})(\mathrm{r}=0.381$; $p=0.080)(D)$. Motor timing errors were significantly correlated with BP and MP, not MMP. BP: Bereitschaftpotential, MP: motor potential, MMP: movement monitoring potential. 
Table 2. Correlations between behavior characteristics and MRP components

\begin{tabular}{lcccc}
\hline & Early BP & Late BP & MP & MMP \\
\hline Age & -0.162 & -0.296 & -0.162 & 0.051 \\
Motor timing errors & $0.485^{*}$ & $0.511^{*}$ & $0.570^{\dagger}$ & 0.381 \\
EMG peak amplitude & -0.188 & -0.203 & -0.079 & -0.155 \\
EMG peak latency & 0.396 & 0.352 & $0.440^{*}$ & 0.176 \\
\hline
\end{tabular}

Values are Pearson's correlation coefficients. ${ }^{*} \mathrm{p}<0.05,{ }^{\dagger} \mathrm{p}<0.01$. MRP: movement-related potential, EMG: electromyogram, BP: Bereitschaftpotential, MP: motor potential, MMP: Movement monitoring potential

Table 3. Linear regression models of late BP amplitude as predicted by behavior characteristics

\begin{tabular}{lcccc}
\hline & $\beta$ & $\mathrm{B}(\mathrm{SE})$ & $\mathrm{t}$ & $\mathrm{p}$ \\
\hline $\begin{array}{l}\text { Model 1 } \\
\quad \text { Constant) }\end{array}$ & & $-2.395(0.319)$ & -7.517 & $<0.001$ \\
$\quad$ Motor timing errors & 0.511 & $-0.001(0.0002)$ & 2.661 & $0.015^{*}$ \\
Model 2 & & & & \\
$\quad$ Constant) & & $-2.580(0.356)$ & -7.257 & $<0.001$ \\
Motor timing errors & 0.448 & $0.0005(0.0002)$ & 2.252 & $0.036^{*}$ \\
EMG peak latency & 0.226 & $0.001(0.001)$ & 1.138 & 0.259 \\
\hline
\end{tabular}

Linear regression analyses were performed with the late $\mathrm{BP}$ as dependent variable, and motor timing errors and EMG latency as independent variables. Standardized regression coefficients $(\beta)$. Understandardized coefficients (B) and standard errors (S.E.). ${ }^{*} \mathrm{p}<0.05$. EMG: electromyogram, BP: Bereitschaftpotential

Table 4. Linear regression models of MP amplitude as predicted by behavior characteristics

\begin{tabular}{lccrc}
\hline & $\beta$ & $\mathrm{B}(\mathrm{SE})$ & $\mathrm{t}$ & $\mathrm{p}$ \\
\hline $\begin{array}{l}\text { Model 1 } \\
\quad \text { Constant) }\end{array}$ & & $-4.232(0.419)$ & -10.102 & $<0.001$ \\
$\quad$ Motor timing errors & 0.570 & $0.001(0.0003)$ & 3.099 & $0.006^{\dagger}$ \\
Model 2 & & & & \\
$\quad$ (Constant) & & $-4.573(0.452)$ & -10.128 & $<0.001$ \\
Motor timing errors & 0.484 & $0.001(0.0003)$ & 2.252 & $0.016^{*}$ \\
EMG peak latency & 0.304 & $0.002(0.001)$ & 1.138 & 0.114 \\
\hline
\end{tabular}

Linear regression analyses were performed with the BP as dependent variable, and motor timing errors and EMG latency as independent variables. Standardized regression coefficients ( $\beta)$. Understandardized coefficients (B) and standard errors (S.E.). ${ }^{*} \mathrm{p}<0.05$, ${ }^{\dagger} \mathrm{p}<0.01$. EMG: electromyogram, MP: motor potential

\section{DISCUSSION}

MRPs are not confined to motor-specific processes, but involve non-motor or cognitive factors. ${ }^{19}$ However, it remains somewhat unclear that internal time-processing is reflected in voluntary movement-related potential. We sought to investigate the motor timing that contributed to self-initiated voluntary movement and to examine how the contribution of mo- tor timing is reflected in movement-related neural activities in a sample of healthy subjects. To the best of our knowledge, this study is the first to examine the pattern of neural activity associated with motor timing processes during voluntary movements for all components of the MRP.

The activation patterns observed in this study were similar to those of previous studies that analyzed MRP as the peak amplitude over the central-medial scalp using EMG as a movement-onset trigger. ${ }^{11}$ During self-triggered movements, the negativities of all MRP components were concentrated around the vertex region. ${ }^{31,32} \mathrm{~A}$ slow negative event-related potential waveform, which spread over time before and after EMG onset, was observed in the surface recorded at the central-located electrodes. The amount of neural activation followed the predicted inverted-U-shaped pattern. ${ }^{7,33}$ The negative potential developed prior to the motor timing response was maximal at its peak and, thereafter, the rebound was observed. ${ }^{3,32}$ A plausible hypothesis to explain these results is that MRPs reflect the integral sum of activity or excitability over the SMA (Cz electrode sites-it has been suggested that the SMA is the origin of the MRP because it is maximally recorded at $\mathrm{Cz}$, which overlies this anatomical structure) during self-initiated movement. ${ }^{34}$

Typical studies on BP restrict the intervals between movements, which could be once every 5 seconds. To measure the rate from the subjective internal clock assumed to be the natural strategy adopted by subjects partaking in motor timing tasks for time intervals of longer than 3 seconds, motor timing tasks in this study required movements to be voluntarily selfinitiated by subjects without external trigger signals. $.^{10} \mathrm{The} \mathrm{du}-$ ration of interval time is a factor that may influence the decrease in MRP intensity, ${ }^{30}$ and perception or processing of shorter and longer time intervals evoke different modes of temporal processing. ${ }^{35}$ In particular, time intervals of less than three seconds are thought to fall within the range of the "perceived present." This is supported by psychophysiological evidence suggesting that the threshold of a duration to be defined as the psychological present-which can be perceived as an entity and therefore is not influenced by working memory-lies between 1 and $3 \mathrm{~s}$. In contrast, longer intervals might activate silent counting or internal clocks as compared with the holistic processing of shorter intervals. ${ }^{35,36}$

In response to the requirement to repeat the same action in a self-initiated manner once every 5 seconds, subjects have an internal temporal guide on when to move. Subjects might set up and rely on some internally generated blurry-defined rhythmical activity. The activity might be initiated periodically and then increase until some subjective threshold is reached, which is determined by the subject. This activity may be represented by the amplitudes of BP to MP. According to this suggestion, an actual movement is executed when random activity has ac- 
cumulated enough power to cross an electrophysiological threshold, indicating that the motor command requires a final trigger to actually generate movement. This explanation may reflect the triggering mechanism for actual movement. ${ }^{8,20,33}$

Upon investigating the impact of the relationship between the degree of movement-related neural activity and the behavior characteristics on motor timing tasks, a significant correlation between the magnitude of the MRP and motor timing errors was found in BP and MP, not MMP. MP also had a significant correlation with EMG latency. However, there was no significant correlation across all MRP components between the EMG amplitude and the MRP amplitude. Notably, as the motor timing error increase, the BP and MP magnitude decrease. To exclude the possibility that the modulation of the MRPs found in our study were induced by differences in muscular activity during movements (for example, force and speed of the movements), a regression analysis was performed with the performance score as an independent variable and the MRP as a dependent variable. Motor timing error was found to have a significant effect on amplitude of late BP and MP. This finding is consistent with the fMRI data in which the motor timing condition was associated with greater activation in the SMA. Relationships between SMA and time estimation have been well-established in fMRI studies. They were also mentioned long ago in studies on the relationship between SMA and $\mathrm{BP}^{37}$

SMA is a crucial region contributing to the sustained activity of the RP before movement. ${ }^{38}$ Our data are consistent with the involvement of SMA in internal time processes during voluntary movement. Several studies have reported that the timecourse of firing of neurons in the pre-motor and motor cortex bore are similar to the time-course of the MRP, and the activation of MFC is required for temporal processing. The postmovement component (MMP) may represent activity, which reactivated after the initial activation peak of the MRP. We expected SMA to play a causal role in introspective motor timing and that the medial frontal cortex (MFC; electrode $\mathrm{Cz}$ from EEG) would be involved in motor timing and timing error detection. However, there was no significant correlation between MMP and the motor timing error.

In this study, the motor timing error contributed independently to the amplitude of the late BP and MP measured at $\mathrm{Cz}$, while the exerted force and speed did not contribute independently to any component. Several studies have shown that BP is influenced by the exerted forces during movement and speed of movement. It is difficult to clearly distinguish the effects of psychological factors from those of physical factors because stronger and faster exertions are generally associated with greater intentions, motivations, and efforts of subjects. MRPs are also found in tasks where subjects only imagine their movement, rather than actually performing any movement, and no motor output is present. RP-like electrophysiological potentials have been identified, regardless of whether the movement is actually performed when subjects form a movement intention. Jan-

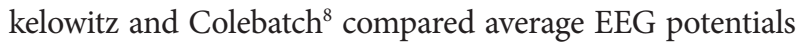
recorded at the central vertex electrode $(\mathrm{Cz})$ during motor imagery and self-initiated movement; they observed equal early BPs in all the conditions. An important question is "how are cortical activities observed by imagination alone without any active motor execution?" In the same vein, brain areas activated by motor imagery were compared to motor execution using fMRI by Cunnington et al. ${ }^{39}$ They found that SMA was active in both motor imagery and motor execution, whereas MI was active only in motor execution.

Control for action is not a single holistic process. The process reflected by MRPs is, of course, not the timing of mechanisms itself. MRPs could encode cognitive demands in addition to temporal information, such as attention, ${ }^{19}$ intention, ${ }^{23,32}$ or working memory. ${ }^{40}$ Accuracy and precision of time estimation and the exact timing of motor behavior are intimately linked to overall cognitive function. Considering the design of this study, it is reasonable to assume that movement preparation and the activation of the internal clock may have occurred simultaneously, and internal clock activation may influence the magnitude of the MRP.

We observed that MRPs recorded during self-initiated movement were significantly different between the first quintile (Q1) and fifth quintile (Q5) of motor timing error with different performance capabilities of motor timing. Specifically, MRP amplitude was significantly attenuated more in Q1 than Q5. The absolute error was 2.63 seconds in Q1 and 0.09 seconds in Q5. The mean interval time of the latter was 5.03 seconds, and the former was 7.62 seconds, with the interval time delayed by 2 seconds. Our results confirmed that internally generated rhythms used as a self-reference for motor timing were slower in Q1 with larger motor timing errors. This could be due to the tendency among subjects in Q1 to overestimate the target interval, thus ending in a directional error. ${ }^{14}$ The current study focused on the link between absolute errors in temporal judgment and the MRP amplitude. However, temporal judgments involve not only absolute but also directional errors. Hence, it remains unclear whether the attenuated mean MRP amplitude was the result of an absolute error or whether it was due to a directional error. This issue is likely due to the small study sample size, which constituted a limitation in the present research.

Furthermore, given the limited design scope of this study, it is not possible to exclude the possibility that subjects might have failed to maintain constant attention during all motor timing tasks or to rule out the potential that counting strategies may have been used by subjects for motor timing. ${ }^{9}$ Not count- 
ing seconds is a classical requirement in BP research, out of concern that subjects may not act by free will. Verleger et al. ${ }^{41}$ investigated the BP between conditions of mental time estimation and conditions of no mental time estimation, but found no difference. They suggested that time tracking would be enabled through several strategies similar to counting in the mind when counting is not required.

Notwithstanding its limitations, the current study demonstrated that increase in the level of motor timing error with the target timing caused a significant attenuation of late BP and MP at the central-midline electrode. Motor timing errors and MRPs measured during motor timing tasks have never been directly compared and therefore remain understudied. The notion of a link between a decrease in MRP negativity and motor timing deficits needs to be explored by further research. The temporal information has to be adequately processed for the control of perception and action. The current study provides further evidence that MRPs are associated with a deficit in the sense of time, at least in that measured by motor timing tasks. The level of the motor timing error with the target timing was found to have an important effect on the late BP and MP amplitude of the MRP. Although these findings are only preliminary, they indicate that motor timing abilities may have a substantial influence on neural activities related to self-initiated voluntary movement. These results may help to improve the understanding of motor timing and its possible role in central motor control processes.

\section{Supplementary Materials}

The online-only Data Supplement is available with this article at https://doi.org/10.30773/pi.2020.0434.

\section{Acknowledgments}

This work was supported by the National Research Foundation of Korea (NRF) grant funded by the Korean government (MSIT) (no. NRF2019R1A 2C4069598).

\section{Conflicts of Interest}

The authors have no potential conflicts of interest to disclose.

\section{Author Contributions}

Conceptualization: Jin Young Park, Jee Seon Ahn. Data curation: Jee Seon Ahn. Formal analysis: Jee Seon Ahn. Funding acquisition: Jin Young Park. Investigation: Jee Seon Ahn, Jun Ho Yoon. Methodology: Jee Seon Ahn. Project administration: Jin Young Park. Resources: Jin Young Park. Software: Jee Seon Ahn. Supervision: Jin Young Park, Jae-Jin Kim. Writing_original draft: Jee Seon Ahn. Writing_review \& Editing: Jee Seon Ahn, Jin Young Park.

\section{ORCID iDs}

Jee Seon Ahn Jun Ho Yoon Jae-Jin Kim

Jin Young Park
https://orcid.org/0000-0002-1294-117X https://orcid.org/0000-0002-6182-5909 https://orcid.org/0000-0002-1395-4562 https://orcid.org/0000-0002-5351-9549

\section{REFERENCES}

1. Shih LY, Kuo WJ, Yeh TC, Tzeng OJ, Hsieh JC. Common neural mechanisms for explicit timing in the sub-second range. Neuroreport 2009; 20:897-901.

2. Duzcu H. A neural marker of the start-gun in interval timing: onset N1P2. Behav Neurosci 2019;133:414-427.

3. Shibasaki H, Hallett M. What is the bereitschaftspotential? Clin Neurophysiol 2006;117:2341-2356.

4. Gaetz M, Bernstein DM. The current status of electrophysiologic procedures for the assessment of mild traumatic brain injury. J Head Trauma Rehabil 2001;16:386-405.

5. Deiber MP, Sallard E, Ludwig C, Ghezzi C, Barral J, Ibañez V. EEG alpha activity reflects motor preparation rather than the mode of action selection. Front Integr Neurosci 2012;6:59.

6. Deecke L, Scheid P, Kornhuber HH. Distribution of readiness potential, pre-motion positivity, and motor potential of the human cerebral cortex preceding voluntary finger movements. Exp Brain Res 1969;7: 158-168.

7. Shibasaki H, Barrett G, Halliday E, Halliday A. Cortical potentials associated with voluntary foot movement in man. Electroencephalogr Clin Neurophysiol 1981;52:507-516.

8. Jankelowitz SK, Colebatch JG. Movement-related potentials associated with self-paced, cued and imagined arm movements. Exp Brain Res 2002;147:98-107.

9. Bortoletto M, Cunnington R. Motor timing and motor sequencing contribute differently to the preparation for voluntary movement. Neuroimage 2010;49:3338-3348.

10. Pastor M, Artieda J, Jahanshahi M, Obeso J. Time estimation and reproduction is abnormal in Parkinson's disease. Brain 1992;115:211-225.

11. Shoushtarian M, Murphy A, Iansek R. Examination of central gait control mechanisms in Parkinson's disease using movement-related potentials. Mov Disord 2011;26:2347-2353.

12. Torta DME, Castelli L, Latini-Corazzini L, Banche A, Lopiano L, Geminiani G. Dissociation between time reproduction of actions and of intervals in patients with Parkinson's disease. J Neurol 2010;257:13561361.

13. Snowden AW, Buhusi CV. Neural correlates of interval timing deficits in schizophrenia. Front Hum Neurosci 2019;13:9.

14. Barkley RA, Murphy KR, Bush T. Time perception and reproduction in young adults with attention deficit hyperactivity disorder. Neuropsychology 2001;15:351-360.

15. Smith A, Taylor E, Rogers JW, Newman S, Rubia K. Evidence for a pure time perception deficit in children with ADHD. J Child Psychol Psychiatry 2002;43:529-542.

16. Isaksson S, Salomäki S, Tuominen J, Arstila V, Falter-Wagner CM, Noreika V. Is there a generalized timing impairment in Autism Spectrum Disorders across time scales and paradigms? J Psychiatr Res 2018;99:111-121.

17. Karp BI, Porter S, Toro C, Hallett M. Simple motor tics may be preceded by a premotor potential. J Neurol Neurosurg Psychiatry 1996; 61:103-106.

18. Thelen E. Timing in motor development as emergent process and product. Adv Psychol 1991;81:201-211.

19. Baker KS, Piriyapunyaporn T, Cunnington R. Neural activity in readiness for incidental and explicitly timed actions. Neuropsychologia 2012;50:715-722.

20. Green JB, StArnold PA, Rozhkov L, Strother DM, Garrott N. Bereitschaft (readiness potential) and supplemental motor area interaction in movement generation: spinal cord injury and normal subjects. J Rehabil Res Dev 2003;40:225-234.

21. Buhusi CV, Meck WH. What makes us tick? Functional and neural mechanisms of interval timing. Nat Rev Neurosci 2005;6:755-765.

22. Di Russo F, Lucci G, Sulpizio V, Berchicci M, Spinelli D, Pitzalis S, et al. Spatiotemporal brain mapping during preparation, perception, and 
action. NeuroImage 2016;126:1-14.

23. Giovannelli F, Mastrolorenzo B, Rossi A, Gavazzi G, Righi S, Zaccara $\mathrm{G}$, et al. Relationship between impulsivity traits and awareness of motor intention. Eur J Neurosci 2016;44:2455-2459.

24. Rossi A, Giovannelli F, Gavazzi G, Righi S, Cincotta M, Viggiano M. Electrophysiological activity prior to self-initiated movements is related to impulsive personality traits. Neuroscience 2018;372:266-272.

25. Wright DJ, Holmes PS, Smith D. Using the movement-related cortical potential to study motor skill learning. J Mot Behav 2011;43:193-201.

26. Hodges PW, Bui BH. A comparison of computer-based methods for the determination of onset of muscle contraction using electromyography. Electroencephalogr Clin Neurophysiol 1996;101:511-519.

27. Delorme A, Makeig S. EEGLAB: an open source toolbox for analysis of single-trial EEG dynamics including independent component analysis. J Neurosci Methods 2004;134:9-21.

28. van der Salm SM, Tijssen MA, Koelman JH, van Rootselaar AF. The bereitschaftspotential in jerky movement disorders. J Neurol Neurosurg Psychiatry 2012;83:1162-1167.

29. Vercillo T, O'Neil S, Jiang F. Action-effect contingency modulates the readiness potential. Neuroimage 2018;183:273-279.

30. do Nascimento OF, Nielsen KD, Voigt M. Influence of directional orientations during gait initiation and stepping on movement-related cortical potentials. Behav Brain Res 2005;161:141-154.

31. Colebatch JG. Bereitschaftspotential and movement-related potentials: Origin, significance, and application in disorders of human movement. Mov Disord 2007;22:601-610.

32. Shakeel A, Navid MS, Anwar MN, Mazhar S, Jochumsen M, Niazi IK. A review of techniques for detection of movement intention using movement-related cortical potentials. Comput Math Methods Med 2015;2015:346217.
33. Macar F, Vidal F, Casini L. The supplementary motor area in motor and sensory timing: evidence from slow brain potential changes. Exp Brain Res 1999;125:271-280.

34. Deecke L, Kornhuber HH. An electrical sign of participation of the mesial 'supplementary' motor cortex in human voluntary finger movement. Brain Res 1978;159:473-476.

35. Elbert T, Ulrich R, Rockstroh B, Lutzenberger W. The processing of temporal intervals reflected by CNV-like brain potentials. Psychophysiology 1991;28:648-655.

36. Rubia K, Halari R, Christakou A, Taylor E. Impulsiveness as a timing disturbance: neurocognitive abnormalities in attention-deficit hyperactivity disorder during temporal processes and normalization with methylphenidate. Philos Trans R Soc Lond B Biol Sci 2009;364:19191931.

37. Deecke L, Kornhuber HH, Lang W, Lang M, Schreiber H. Timing function of the frontal cortex in sequential motor and learning tasks. Hum Neurobiol 1985;4:143-154.

38. Nguyen VT, Breakspear M, Cunnington R. Reciprocal interactions of the SMA and cingulate cortex sustain premovement activity for voluntary actions. J Neurosci 2014;34:16397-16407.

39. Cunnington R, Windischberger C, Moser E. Premovement activity of the pre-supplementary motor area and the readiness for action: studies of time-resolved event-related functional MRI. Hum Mov Sci 2005;24: 644-656.

40. Brown SW. Attentional resources in timing: interference effects in concurrent temporal and nontemporal working memory tasks. Percept Psychophys 1997;59:1118-1140.

41. Verleger R, Haake M, Baur A, Śmigasiewicz K. Time to move again: does the bereitschaftspotential covary with demands on internal timing? Front Hum Neurosci 2016;10:642. 
Supplementary Table 1. The number of ICA components and trials in EEG artifact removal

\begin{tabular}{|c|c|c|c|c|c|c|}
\hline \multirow{2}{*}{ Subjects } & \multicolumn{3}{|c|}{ ICA component } & \multicolumn{3}{|c|}{ Trials } \\
\hline & Total & Left & Removal & Total & Left & Removal \\
\hline Subjects 1 & 65 & 61 & 4 & 44 & 43 & 1 \\
\hline Subjects 2 & 65 & 59 & 6 & 51 & 43 & 8 \\
\hline Subjects 3 & 65 & 62 & 3 & 97 & 57 & 40 \\
\hline Subjects 4 & 65 & 59 & 6 & 99 & 96 & 3 \\
\hline Subjects 5 & 65 & 57 & 8 & 73 & 58 & 15 \\
\hline Subjects 6 & 65 & 62 & 3 & 60 & 53 & 7 \\
\hline Subjects 7 & 65 & 59 & 6 & 39 & 36 & 3 \\
\hline Subjects 8 & 65 & 57 & 8 & 63 & 62 & 1 \\
\hline Subjects 9 & 65 & 62 & 3 & 51 & 51 & 0 \\
\hline Subjects 10 & 65 & 62 & 3 & 59 & 57 & 2 \\
\hline Subjects 11 & 65 & 56 & 9 & 41 & 40 & 1 \\
\hline Subjects 12 & 65 & 57 & 8 & 66 & 66 & 0 \\
\hline Subjects 13 & 65 & 61 & 4 & 69 & 63 & 6 \\
\hline Subjects 14 & 65 & 61 & 4 & 60 & 55 & 5 \\
\hline Subjects 15 & 65 & 59 & 6 & 77 & 69 & 8 \\
\hline Subjects 16 & 65 & 58 & 7 & 66 & 60 & 6 \\
\hline Subjects 17 & 65 & 58 & 7 & 66 & 64 & 2 \\
\hline Subjects 18 & 65 & 56 & 9 & 46 & 45 & 1 \\
\hline Subjects 19 & 65 & 60 & 5 & 57 & 52 & 5 \\
\hline Subjects 20 & 65 & 59 & 6 & 70 & 68 & 2 \\
\hline Subjects 21 & 65 & 61 & 4 & 73 & 68 & 5 \\
\hline Subjects 22 & 65 & 58 & 7 & 68 & 65 & 3 \\
\hline
\end{tabular}




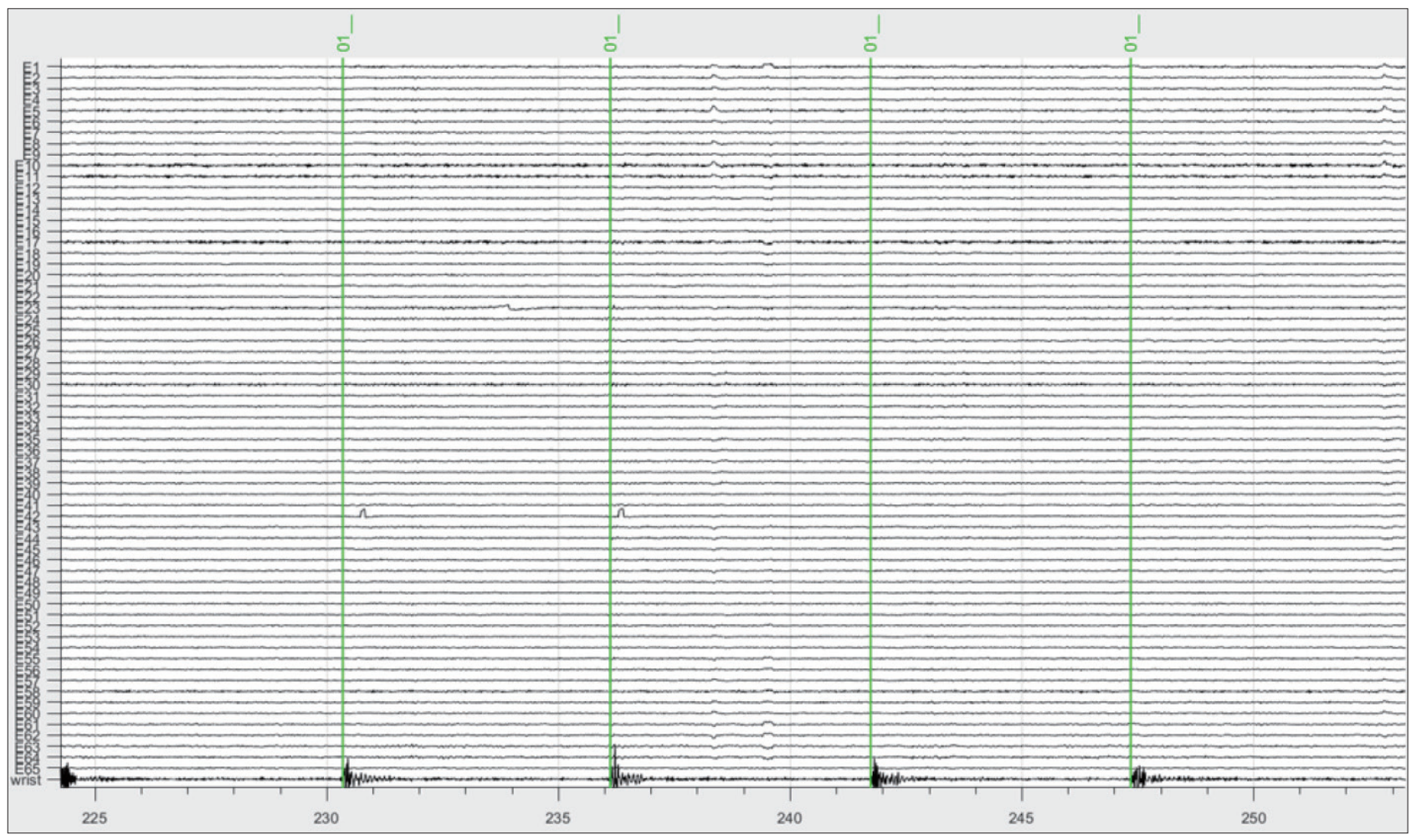

Supplementary Figure 1. Simultaneous scalp EEG (E1-E65) and EMG (wrist) recording. Time intervals between consecutive EMG onsets performed. X-axis: time, Y-axis: signal amplitude, one row per channel. Events are displayed green line. EEG: electroencephalogram, EMG: electromyogram. 